\title{
Lemon Juice: A potential source of Angiotensin Converting Enzyme antagonism for weight loss and insulin resistance
}

\author{
S. Tejpal ${ }^{1}$, C. Bastie ${ }^{1}$ and J. Klein Seetharaman ${ }^{1,2}$ \\ ${ }^{1}$ Division of Biomedical Sciences, Warwick Medical School, University of Warwick, Coventry, CV4 7AL, UK and \\ ${ }^{2}$ Institute for Digital Healthcare, Warwick Manufacturing Group, University of Warwick, Coventry, CV4 7AL, UK.
}

Being overweight and/obese is associated with metabolic disorders such as diabetes and insulin resistance ${ }^{(1)}$. Angiotensin Converting Enzyme (ACE) has been identified as a potential marker for weight loss ${ }^{(2)}$. The mechanisms by which nutrients regulate molecular pathways involved in adipose tissue metabolism have recently become of high interest ${ }^{(3)}$. Lemon extracts appear to improve weight loss and reduce ACE activity ${ }^{(3)}$. Here, we aim to understand the potential use of ACE as an early marker for weight loss and determine whether lemon extracts effects ACE gene expression and insulin sensitivity in adipocytes.

52 participants ( $77 \%$ females and $23 \%$ males; mean BMI $27 \pm 5$ ) were instructed to follow a 1200 Kcal diet with an optional daily $<250 \mathrm{Kcal}$-snack. They used an in-house generated health platform to provide urine and diaries of food intake and physical activity. A total of 149 days information was recorded by the participants on the health platform. The study was approved by Warwick Medical School Ethics committee BSREC. ACE concentration was measured using a Human ACE DuoSet Elisa from R\&D System. 3T3L1 adipocytes were differentiated as previously described in $^{(4)}$ and treated with lemon extract (LJ). ACE mRNA expression was quantified by qPCR using Taqman gene expression assays. PhosphoAKT (p-AKT) protein levels were used to assess insulin responsiveness in $3 \mathrm{~T} 3 \mathrm{~L} 1$ cells treated with $100 \mu \mathrm{g} / \mathrm{ml} \mathrm{LJ}$ and $100 \mathrm{nM}$ insulin. Pearson correlation and Mann Whitney U test were performed using R to understand correlation between ACE and weight change parameters.

We found that following a day of dieting (so far without lemon juice), ACE positively correlated with weight difference; reduction was significantly more robust in individuals with a BMI $>25(\mathrm{p}<0.05$ and $\mathrm{p}<0.005$ respectively; Fig 1a, b). ACE gene expression significantly increased (up to 12-fold at day 8) during 3T3L1 differentiation (Fig 1c). Addition of LJ on differentiated cells led to an $80 \%$ reduction in ACE gene expression in adipocytes (Fig 1d). Consistent with this, LJ-induced ACE reduction was associated with increased insulin sensitivity in adipocytes, evidenced by increased p-AKT levels (Fig 1e).

(a)

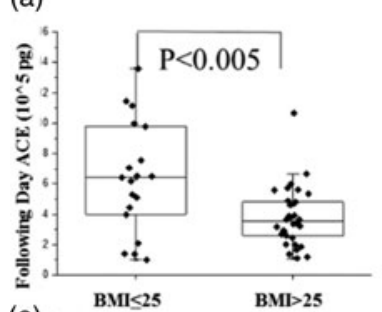

(e) 5 (b)

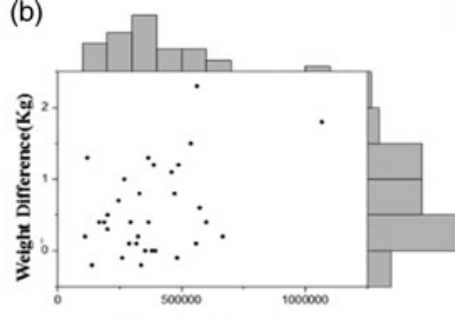

Following Day ACE (pg)

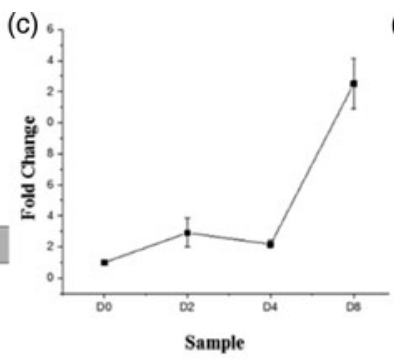

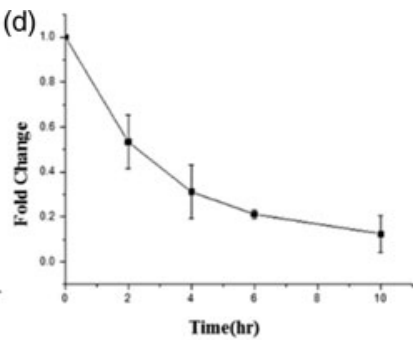

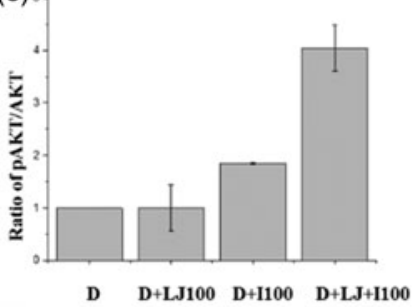

Fig. 1. (a) ACE response is dependent upon BMI. (b) Following day ACE is positively related to weight difference. (c) ACE mRNA expression during 3T3L1 adipocytes differentiation.(d) Effects of lemon juice LJ on ACE mRNA expression in adipocytes.(e) Phospho-AKT and total AKT levels change with treatment of $100 \mathrm{ug} / \mathrm{ml} \mathrm{LJ}$ after $10 \mathrm{~h}$ followed by incubation with/without $100 \mathrm{~nm}$ insulin(I).

In summary, our studies demonstrate that, ACE levels correlate with BMI and weight loss as early as after only 1 day of dieting, and thus could be a potential early "biofeedback" marker on dieting and weight loss. LJ reduction in ACE expression has been found in a model of adipocytes and this is also associated with increased insulin sensitivity.

1. Smith A \& Singleton (2013) J Diabetes Complicat 27, 436-442.

2. Wang P, Holst C, Andersen M, Astrup A et al. (2011) PLoS ONE 6, e16773.

3. Oboh G, Bello F, Ademosun A et al. (2015) Comp Clin Path 24, 1395-1406.

4. Zebisch K, Voigt V, Wabitsch M et al. (2012) Anal Biochem 425, 88-90. 